\title{
Metallomics
}

Check for updates

Cite this: Metallomics, 2017 9, 981

Received 5th April 2017 Accepted 21st June 2017 DOI: $10.1039 / \mathrm{c} 7 \mathrm{mt} 00101 \mathrm{k}$

rsc.li/metallomics

\section{Probing functional roles of Wilson disease protein (ATP7B) copper-binding domains in yeast $\dagger$}

\author{
Kumaravel Ponnandai Shanmugavel, ${ }^{a}$ Dina Petranovic ${ }^{\mathrm{ab}}$ and \\ Pernilla Wittung-Stafshede (D) *a
}

\begin{abstract}
After Ctr1-mediated uptake into human cells, copper $(\mathrm{Cu})$ ions are transported by the cytoplasmic $\mathrm{Cu}$ chaperone Atox1 to the Wilson disease protein (ATP7B) in the Golgi network. Cu transfer occurs via direct protein-protein interactions and leads to incorporation of $\mathrm{Cu}$ into $\mathrm{Cu}$-dependent enzymes. ATP7B is a large multi-domain membrane-spanning protein which, in contrast to homologs, has six cytoplasmic metal-binding domains (MBDs). The reason for multiple MBDs is proposed to be indirect modulation of activity but mechanistic studies of full-length ATP7B are limited. We here developed a system that probes $\mathrm{Cu}$ flow through human Atox1 and ATP7B proteins when expressed in yeast. Using this assay, we assessed the roles of the different MBDs in ATP7B and found that the presence of the most $\mathrm{N}$-terminal MBD increased, whereas the third MBD decreased, overall ATP7B-mediated $\mathrm{Cu}$ transport activity. Upon removal of all MBDs in ATP7B, the ability to transport Cu disappeared. The designed system can be expanded to include other yeast viability parameters and will be a useful tool for further mechanistic insights on human Cu transport as well as diseases involving Cu imbalance.
\end{abstract}

\section{Significance to metallomics}

Copper is an essential metal ion facilitating many biological functions but can also promote human disease at certain conditions. To avoid toxicity of free copper ions, there are elaborate protein-based transport systems for copper ions in cells that deliver the metal to target proteins. Many questions around copper transport proteins, which includes soluble chaperones and large multi-domain membrane-bound proteins, remain unanswered, in part because of a lack of structural information as these proteins are often hard to purify. We here developed a yeast system that probes copper flow through the human cytoplasmic copper transport proteins, Atox1 and ATP7B, without the need for purification and we used the system to probe aspects of ATP7B's unique metal-binding domains. This new tool can be expanded to probe a range of mechanistic questions of copper transport, for identification of unknown proteins involved in copper transport, as well as for investigations of human diseases involving $\mathrm{Cu}$ misbalance (such as genetic diseases, cancer and neurodegeneration).

\section{Introduction}

Copper $(\mathrm{Cu})$ ions in oxidized and reduced forms are found in the active sites of many essential proteins that participate in key cellular reactions. ${ }^{1-3}$ However, free $\mathrm{Cu}$ ions are potentially toxic for cells since, due to their redox activity, they are capable of producing reactive oxygen species. ${ }^{4}$ To avoid $\mathrm{Cu}$ toxicity, the intracellular concentration of $\mathrm{Cu}$ is regulated via dedicated proteins that facilitate uptake, efflux as well as distribution of $\mathrm{Cu}$ (in its reduced form) to $\mathrm{Cu}$-dependent proteins and

\footnotetext{
${ }^{a}$ Department of Biology and Biological Engineering, Chalmers University of Technology, 41296 Gothenburg, Sweden. E-mail: Pernilla.wittung@chalmers.se

${ }^{b}$ Novo Nordisk Foundation, Center for Biosustainability, Chalmers University of Technology, 412 96, Gothenburg, Sweden

$\dagger$ Electronic supplementary information (ESI) available: Tables S1-S4 and Fig. S1-S4. See DOI: 10.1039/c7mt00101k
}

enzymes. ${ }^{5-7}$ In the human cytoplasm, after the uptake of $\mathrm{Cu}$ ions by the membrane-spanning Ctr1 trimer, ${ }^{8}$ the soluble, single-domain $\mathrm{Cu}$ chaperone Atox1 transports the metal to ATP7A and ATP7B (also called Menke's and Wilson disease proteins, respectively), two homologous membrane-bound $\mathrm{P}_{1 \mathrm{~B}}$-type ATPases located in the trans-Golgi network. Once transferred to ATP7A/B, the $\mathrm{Cu}$ ion is channeled through the protein to the lumen of the Golgi where it is loaded onto specific $\mathrm{Cu}$-dependent proteins and enzymes such as lysyl oxidase and ceruloplasmin.

ATP7A/B are large (about 1400 residues), multi-domain proteins with several domains protruding in the cytoplasm as well as membrane spanning parts that harbors a channel for $\mathrm{Cu}$. Unique for ATP7/B are six cytoplasmic metal-binding domains (MBDs; named MBD1 to MBD6, with MBD1 being the first from the N-terminus and MBD6 the domain closest to the membranespanning part of ATP7A/B) connected by peptide linkers of various lengths constituting the N-terminal tail. ${ }^{9}$ Each MBD in ATP7A/B, 
as well as Atox1, has a ferredoxin-like $\alpha / \beta$ fold and a surfaceexposed invariant $\mathrm{CXXC}$ motif $(\mathrm{X}=$ any residue) in which a single $\mathrm{Cu}$ can bind to the cysteine sulfurs. In contrast to the human ATP7A/B, corresponding bacterial and yeast $\mathrm{P}_{1 \mathrm{~B}}$-type ATPases have only one or two MBDs. The reason for the presence of multiple MBDs in ATP7A/B has been proposed to involve regulation of $\mathrm{Cu}$ transfer to the Golgi lumen and $\mathrm{Cu}$-mediated protein trafficking between the Golgi and the plasma membrane. ${ }^{10-13}$ During the catalytic cycle which requires ATP hydrolysis and transient phosphorylation, ATP7A/B are likely to undergo significant conformational changes and alterations in domain-domain interactions. ${ }^{9}$ Since there is no high-resolution structural information on the arrangement of the six MBDs within full length ATP7A/B, it is unclear how these domains are arranged relative to each other at different stages of the catalytic cycle. Because Atox1 can deliver $\mathrm{Cu}$ to the MBDs, ${ }^{14-20}$ through direct protein-protein interactions, ${ }^{21,22}$ one may speculate that Cu-triggered conformational changes among these domains might initiate the catalytic cycle after Atox1mediated $\mathrm{Cu}$ delivery. ${ }^{23}$ Nonetheless, it remains unclear if $\mathrm{Cu}$ is being transferred via the MBDs or if Atox1, like its bacterial homolog CopZ, ${ }^{24}$ delivers $\mathrm{Cu}$ directly to a binding site at the membrane-spanning parts of ATP7A/B.

It has been shown in vitro that several MBDs can be deleted/ mutated without loss of $\mathrm{Cu}$ transport activity, but the presence of at least one MBD appears to be required. ${ }^{13,25}$ To explore MBD domain-domain interactions in ATP7B, we have previously investigated several multi-MBD constructs and, through in vitro and in silico analyzes, identified correlated domain-domain structural dynamics in two-domain constructs, ${ }^{26}$ linked domaindomain interactions in WD56 to thermal stability, Cu binding and Atox1-mediated $\mathrm{Cu}$ transfer, ${ }^{27}$ and discovered that $\mathrm{Cu}$ binding triggers compaction of MBD1-4. ${ }^{23}$ However, the studies with truncated ATP7B constructs ca only indirectly address in vivo mechanisms.

One way to circumvent purification of full-length ATP7A/B (which, to date, has not been reported) involves the design of a complementation assay in yeast. ${ }^{28}$ This concept builds on the fact that yeast requires $\mathrm{Cu}$ as cofactor in Fet3p (the yeast homolog of human ceruloplasmin) to survive in iron-limiting conditions. Fet3p is a ferro- $\mathrm{O}_{2}$-oxidoreductase that oxidizes ferrous to ferric iron ions, which are then taken up by the transmembrane permease Ftr1..$^{13,25,29}$ Fet3p obtains its $\mathrm{Cu}$ ions from the pathway involving the yeast Atox1 homolog, Atx1p, and the yeast ATP7A/B homolog, CCC2p. In previous studies, the yeast gene CCC2 was deleted which resulted in reduced growth on iron-limited media. The human ATP7B gene was cloned on a high copy plasmid to complement the phenotype and restore yeast growth on iron-limited media. Using this approach, several point-mutations and MBD truncations/ substitutions in human ATP7B have been investigated. ${ }^{13,29-31}$

Because Atox1 delivers $\mathrm{Cu}$ to ATP7B via direct interactions but the interaction site is unknown, we here created a more advanced yeast system that includes the deletion of both yeast genes (Atx1 and CCC2) with complementation of both human proteins (Atox1 and ATP7B). Thus, in contrast to previous systems, our system retains the natural interactions that governs Cu delivery to ATP7B in humans. First, we demonstrate that the new yeast complementation assay restores the phenotype on iron-limited media. Then we use it to assess functional roles of the six MBDs in ATP7B in the presence of Atox1. Notably, this assay can be extended to numerous mutated Atox1/ATP7B variants to address specific mechanistic questions, and to search for suppressor or enhancer mutations that can guide discovery of new genes involved in human $\mathrm{Cu}$ transport.

\section{Materials and methods}

\section{Cultivation of yeast strains}

Yeast Saccharomyces cerevisiae CEN.PK 113-11C (MATa SUC2 MAL2-8 URA3-52 HIS3- $\Delta 1$ ) was used as reference strain (Dr P. Kötter, Institute of Microbiology, Johann Wolfgang GoetheUniversity, Frankfurt, Germany). The strain has a mutation in CYR1 (codes for adenylate-cyclase and generates cAMP) which leads to high stress resistance during active fermentation and growth. Yeast strains were cultivated on YEPD/YPD (Yeast Extract Peptone Dextrose) media.

\section{Yeast growth rates}

A single yeast colony from plates was inoculated in Fe-limited medium (see below) and incubated overnight at $30^{\circ} \mathrm{C}$ and $200 \mathrm{rpm}$. Next, yeast cells from this culture were washed with ice cold deionized water and cultivated in fresh Fe-limited medium at an initial cell density of $\mathrm{OD}_{600}=0.1$. The growth of the cells at $30{ }^{\circ} \mathrm{C}$ was monitored spectroscopically for 24 hours and growth rates determined from the exponential phase..$^{25}$

\section{Cultivation of $E$. coli}

E. coli was used for plasmid constructions. The E. coli $\mathrm{DH} 5 \alpha$ strain was cultivated on Luria-Bertani (LB) broth medium (10 $\mathrm{g} \mathrm{L}^{-1}$ tryptone, $5 \mathrm{~g} \mathrm{~L}^{-1}$ yeast extract, $10 \mathrm{~g} \mathrm{~L}^{-1} \mathrm{NaCl}$ ), or on plates with the same composition with the addition of $20 \mathrm{~g} \mathrm{~L}^{-1}$ agar. The preparation of competent $E$. coli cells and transformations were carried out according to standard protocols. ${ }^{32}$ Ampicillin was added when required $\left(80 \mu \mathrm{g} \mathrm{mL} L^{-1}\right)$.

\section{Construction of CCC2 and Atx1 yeast deletion strains}

The yeast homologs of human ATP7B and Atox1 genes, CCC2 and Atx1, are located on chromosomes IV and XIV of $S$. cerevisiae, respectively. The $\Delta \mathrm{CCC} 2, \Delta \mathrm{Atx} 1$ and $\Delta \mathrm{CCC} 2 \Delta \mathrm{Atx} 1 S$. cerevisiae strains were constructed by deletion of CCC2 and Atx1 genes individually, and in combination. Deletions of CCC2 and Atx1 genes were performed by replacing the gene with a KanMX deletion cassette which carries the kanamycin selectable marker. The double-deletion strain was constructed by first replacing the CCC2 gene with a KanMX deletion cassette followed by disruption of the kanamycin marker and then replacing the other (Atx1) gene with the KanMX cassette. The KanMX deletion cassette consists of loxP sites flanking both ends of the kanamycin marker. ${ }^{33}$ Disruption of kanamycin was introduced by transforming with a Crelox plasmid pSH47 (Ura- marker, Cre gene, GAL1 promoter) ${ }^{34}$ which integrates at the loxP sites on both ends of the marker via 
the expressed Cre recombinase when grown in galactose medium. Elimination of the kanamycin marker was confirmed, followed by removal of cells containing the pSH47 plasmid by plating on 5-fluoroorotic acid. All deletion strains and modifications were constructed by standard PCR techniques and homologous recombination. DNA primers used for strain constructions are listed in Table S1 (ESI $\dagger)$.

\section{Plasmid constructions}

The human ATP7B and Atox1 gene were cloned in separate plasmids. The plasmids p426GPD (ATCC 87361; GPD, promoter for expression; Ura3 and ampR, markers) and p423GPD (ATCC 87355; GDP, promoter for expression; His3 and ampR, markers) were used as vector plasmids for ATP7B and Atox1 genes, respectively. The ATP7B sequence was extracted from a plasmid from Dr A Koc, ${ }^{35}$ whereas the Atox 1 gene was obtained from a plasmid designed in our lab. ${ }^{22}$ The ATP7B (including constructs with deleted MBDs or mutated variants) and Atox1 genes were amplified with BamHI and SalI restriction sites. Created ATP7B modified constructs were as follows: domain deletions of one MBD to all six MBDs in the ATP7B gene (i.e., ATP7B 1DEL, ATP7B 1-2DEL, ATP7B 1-3DEL, ATP7B 1-4DEL, ATP7B 1-5DEL, and ATP7B 1-6DEL) and point-mutations in the ATP7B gene (i.e., ATP7B CPC-to-SPS, with two Cys replaced with Ser in transmembrane domain 6, and D1027A, with the transiently phosphorylated Asp1027 replaced by Ala). All plasmids were constructed using standard PCR techniques and checked by sequencing (Eurofins Genomics). DNA primers used for plasmid constructions are listed in Table S1 (ESI $\dagger$ ). Plasmids were chemically transformed into yeast cells by the standard lithium-acetate method ${ }^{36}$ and grown on selective media: SD-Urafor the p426GPD vector, SD-His- for the p423GPD vector and SD-Ura-His- for yeast with both plasmids. Constructed yeast strains are listed in Table S2 (ESI $\dagger$ ).

\section{Protein extraction and western blotting}

All yeast strains were grown in iron limited medium for 24 hours at $30{ }^{\circ} \mathrm{C}$. Cell pellets were collected by centrifugation at $2000 \times g$ at $4{ }^{\circ} \mathrm{C}$ for $10 \mathrm{~min}$, washed twice with ice-cold water and lysed with glass beads (lysis buffer: $50 \mathrm{mM}$ HEPES pH 7.5, $150 \mathrm{mM}$ $\mathrm{NaCl}, 2.5 \mathrm{mM}$ EDTA, $1 \% \mathrm{v} / \mathrm{v}$ Triton X100 and freshly added protease inhibitor). After lysis, membranes were collected by centrifugation at $18000 \times g\left(4{ }^{\circ} \mathrm{C}, 30 \mathrm{~min}\right)$. Samples were re-suspended in SDS loading buffer $(0.5 \mathrm{M}$ Tris-HCl, $\mathrm{pH} 6.8$, $10 \%$ SDS, $0.5 \%$ (w/v) bromophenol blue, $87 \%$ glycerol, $100 \mathrm{mM}$ DTT) and $50 \mu \mathrm{g}$ of membranes were loaded on a $4-12 \%$ Bis-Tris gel (Invitrogen) and blotted onto PVDF membranes. ATP7B and Atox1 were detected with monoclonal rabbit ATP7B and Atox1 antibodies, respectively (Abcam, 1:1000 dilution), upon incubation overnight, $4{ }^{\circ} \mathrm{C}$. Next, blots were incubated with horseradish peroxidase conjugated anti-rabbit IgG reagent (Thermo Scientific Pierce) for $15 \mathrm{~min}, 4{ }^{\circ} \mathrm{C}$. Bands were detected by Pierce ${ }^{\mathrm{TM}}$ Fast Western Blot Kits, SuperSignal ${ }^{\mathrm{TM}}$ West Femto, Rabbit (Thermo Scientific Pierce) and visualized with a BioRad ChemiDoc XRS image analyzer.

\section{Yeast complementation assay}

A synthetic defined (SD) medium containing $1.7 \mathrm{~g} \mathrm{~L}^{-1}$ yeast nitrogen base lacking $\mathrm{Fe}$ and $\mathrm{Cu}$ ions, $50 \mathrm{mM}$ MES buffer $\mathrm{pH}$ 6.1, $20 \mathrm{~g} \mathrm{~L}^{-1}$ glucose, $5 \mathrm{~g} \mathrm{~L}^{-1}$ ammonium sulfate was used in combination with a complete supplement mixture (CSM) with dropout as follows. CSM with -Ura and -His dropout was used for the strain carrying both ATP7B and Atox1 plasmids, whereas CSM with -Ura dropout was used for the strain with the ATP7B plasmid and CSM with -His dropout was used for the strain with the Atox1 plasmid. The Fe-limited medium was prepared from the SD medium with the addition of $1 \mathrm{mM}$ ferrozine (Fe chelator), $1 \mu \mathrm{M} \mathrm{CuSO}_{4}$ and $100 \mu \mathrm{M} \mathrm{FeSO}_{4}$. Fe-Supplemented medium was prepared without ferrozine and $350 \mu \mathrm{M} \mathrm{FeSO}_{4}$ and $500 \mu \mathrm{M} \mathrm{CuSO}_{4}$. For the plating assay, transformants were grown in SD medium with $20 \mathrm{~g} \mathrm{~L}^{-1}$ agar. Yeast cells from pre-grown overnight cultures $\left(30{ }^{\circ} \mathrm{C}, 200 \mathrm{rpm}\right)$ were diluted to an optical density at $600 \mathrm{~nm}$ of 0.1 , and then serial dilutions with sterile water were performed followed by plating in Fe-limited and Fe-supplemented medium and incubation at $30{ }^{\circ} \mathrm{C}$ for 3 days. All complementation experiments were carried out at identical conditions $^{25}$ and in duplicates.

\section{Results}

First, we created a yeast strain in which the yeast genes for CCC2p and Atx1p proteins were deleted (Fig. 1A). As expected, the strain $\Delta \mathrm{CCC} 2 \Delta \mathrm{Atx} 1$ did not survive in iron-limiting media (Fig. 2A). In this background, we expressed the human Atox1 and ATP7B genes on high copy plasmids in order to test for complementation and thereby rescue of the phenotype. Western blot analysis confirmed protein expression from the high copy plasmids (Fig. S1A, ESI $\dagger$ ). The $\Delta$ CCC2 $\Delta$ Atx1 yeast strain complemented with Atox1 and ATP7B on plasmids grew in iron-limiting conditions (Fig. 2A). The cell growth rate data (Fig. 2B, Table S3, ESI $\dagger$ ) showed that deletion of one yeast gene and complementing with the corresponding human gene on a plasmid restored normal growth rate, whereas for the double deletion of both CCC2 and Atx1 genes, two plasmids (with Atox1 and ATP7B) were required to restore growth on Fe-limited media (examples of complete growth curves shown in Fig. S2, ESI $\dagger$ ).

To assess the role of the six MBDs in ATP7B Cu-transport function with this system, we created a set of ATP7B domaindeleted variants (Fig. 1B) in which, one-by-one, the MBDs had been removed starting from the $\mathrm{N}$-terminus. We also created 'negative controls' which involved ATP7B genes with (a) a CPCto-SPS mutation in the membrane-spanning $\mathrm{Cu}$ channel and (b) a D1027A mutation removing the residue transiently phosphorylated during the catalytic cycle (Fig. 1B). Both these mutations introduced into ATP7B have been shown previously to block $\mathrm{Cu}$ transport by $\mathrm{ATP} 7 \mathrm{~B} .{ }^{30,37}$ Western blot analysis confirmed that all ATP7B constructs were expressed as proteins in yeast, and at in similar amounts as wild-type ATP7B expression (Fig. S1B, ESI $\dagger$ ). Previous work in a $\triangle \mathrm{CCC} 2$ yeast strain also reported that ATP7B variants added on plasmids expressed protein in similar or higher amounts as wild-type ATP7B; ${ }^{13}$ in 


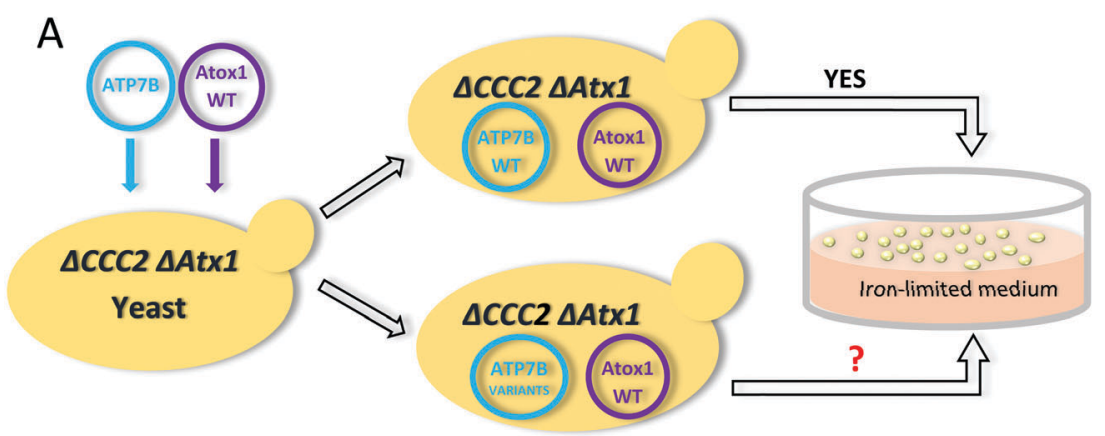

B

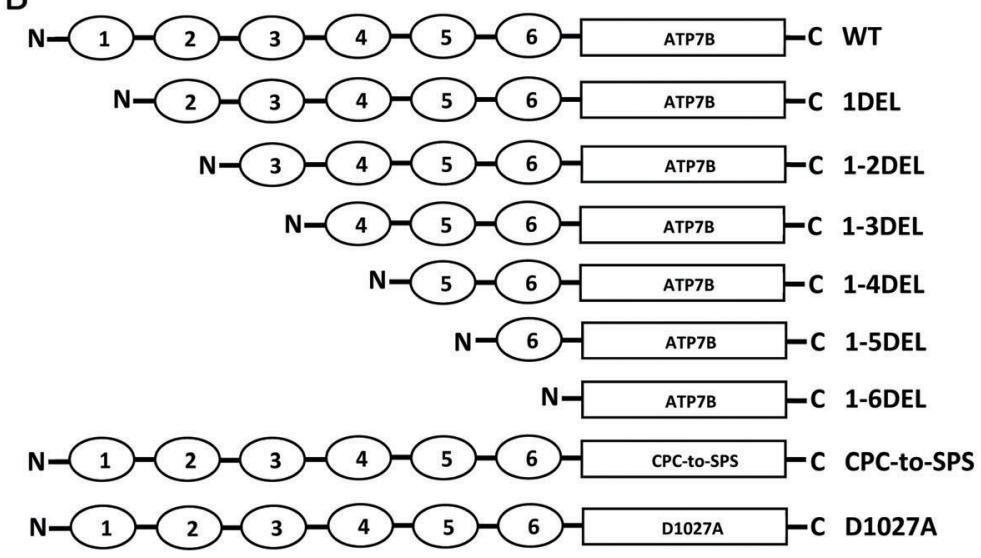

Fig. 1 (A) Schematic representation of the double deletion yeast system designed for human Cu transport studies. Plasmids with human ATP7B and Atox1 were used to complement the yeast strains in which yeast endogenous CCC2 and Atx1 genes were deleted individually or in combination. In this system, we have investigated the consequences of wild type, mutated and truncated ATP7B by probing cell growth on Fe-limited media which requires functional Cu transport. (B) Overview of mutations and MBD deletion constructs of ATP7B that were introduced in the engineered yeast strains.

addition, only small amounts of expressed ATP7B is likely needed to sustain Fet3p activity. As expected, complementing with the two negative control ATP7B genes on plasmids together with the Atox1 plasmid to the $\Delta \mathrm{CCC} 2 \Delta \mathrm{Atx} 1$ yeast strain resulted in growth rates in Fe-limited media corresponding to $\Delta \mathrm{CCC} 2 \Delta \mathrm{Atx} 1$ yeast lacking added ATP7B gene at all and, thus, there was no ATP7Bmediated $\mathrm{Cu}$ transport activity (Fig. 3, Table 1).

Next, growth rates for the $\Delta \mathrm{CCC} 2 \Delta \mathrm{Atx} 1$ yeast strain with the different domain-truncated ATP7B variants were tested, all in combination with wild-type Atox1 and at Fe-limited conditions (Fig. 3, Table S4, ESI $\dagger$ ). Upon comparing yeast with full-length ATP7B to the system with the ATP7B variant in which the first MBD domain has been removed (ATP7B 1DEL), both with wildtype Atox1, yeast growth was reduced by about 70\% (Table 1). However, removing both MBD1 and MBD2 in ATP7B, showed a similar growth rate as upon only removing MBD1 in ATP7B. In contrast, when the first three domains are removed in ATP7B (ATP7B 1-3DEL), wild-type-like growth of $\Delta$ CCC2 $\Delta$ Atx1 yeast was restored. The yeast growth rates were rather similar, 80-90\% of wild-type ATP7B, also upon removal of domains 1-4 (ATP7B 14DEL) and domains 1-5 in ATP7B (ATP7B 1-5DEL), the latter thus with only MBD6 remaining in ATP7B (Fig. 3, Table 1).

Removing all six MBD domains in ATP7B (ATP7B 1-6DEL) resulted in yeast growth comparable to the growth rate of the negative controls, implying that one MBD (only MBD6 tested as a one-MBD ATP7B construct here) is required for ATP7B-mediated
Cu transport activity (Table 1). Notably, when we performed the yeast growth experiments with the domain-truncated ATP7B variants in the absence of the Atox1 plasmid but still in the $\Delta \mathrm{CCC} 2 \Delta \mathrm{Atx} 1$ yeast strain, all strains were similar to negative controls in Fe-limited media (Fig. S3, Table S4, ESI $\dagger$ ). This demonstrated that, in the absence of Atx1p, human Atox1 was essential in providing $\mathrm{Cu}$ to ATP7B.

Next we used the $\triangle \mathrm{CCC} 2$ yeast strain, without complementing with an Atox1 plasmid, to assess the effects on growth by the different domain-truncated ATP7B variants when in the presence of yeast Atx1p, instead of human Atox1 (Fig. S4, Table S4, ESI $\dagger$ ). As Atx1p and human Atox1 may interact differently with human ATP7B, and such an interaction may limit overall $\mathrm{Cu}$ transport, this is an important question we could address with our system. Interestingly, alterations in growth rates for the different domaintruncated ATP7B variants followed the same pattern in Fe-limited media regardless if Atx1 ( $\triangle \mathrm{CCC} 2$ strain, no Atox1 plasmid) or Atox1 ( $\triangle \mathrm{CCC} 2 \Delta \mathrm{Atx} 1$ strain with Atox1 plasmid) acted as the $\mathrm{Cu}$ delivering chaperone ( $c f$. Fig. 3 and Fig. S4, ESI $\dagger$ ).

\section{Discussion}

The yeast $S$. cerevisiae provides an excellent model with which to characterize $\mathrm{Cu}$ delivery to the secretory pathway in further detail because the proteins involved in $\mathrm{Cu}$ homeostasis are 

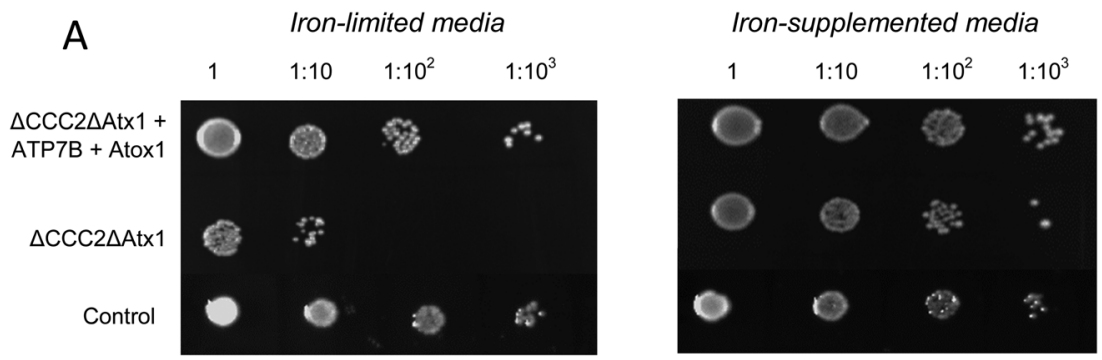

\section{B}

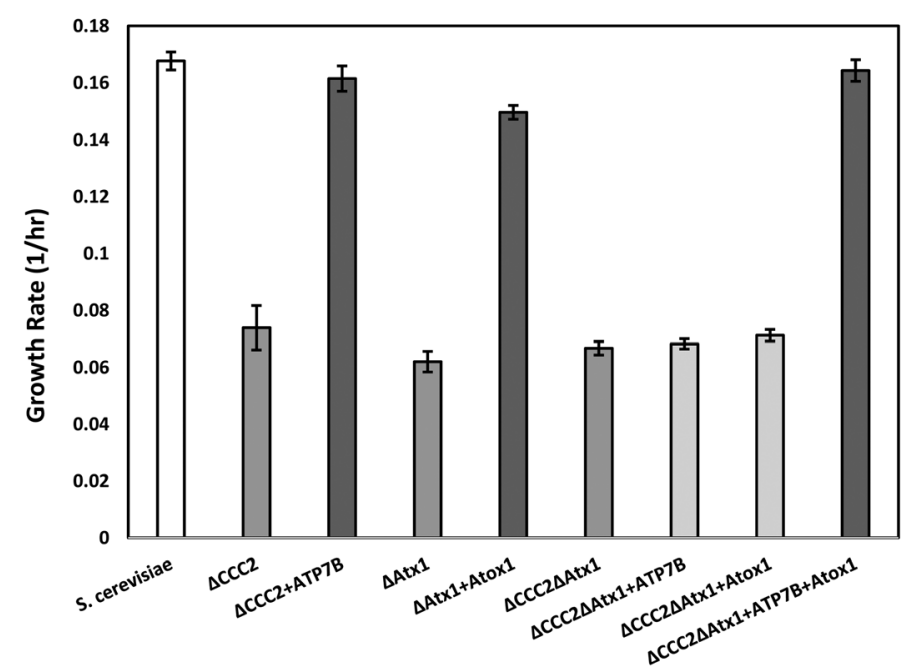

Fig. 2 (A) Plating assay showing serial decimal dilutions of $\triangle C C C 2 \Delta A t \times 1$ yeast cells, without and with added Atox1 and ATP7B plasmids, as well as control (wild-type) yeast, spotted onto Fe-limited and Fe-supplemented media. (B) Growth rates for $\Delta C C C 2, \Delta$ Atx1, $\Delta C C C 2 \Delta$ Atx1 yeast strains complemented with ATP7B and Atox1 plasmids, as described in figure, in Fe-limited conditions (growth rate values reported in Table S3, ESI†). Error bars are based on standard deviation for duplicate experiments.

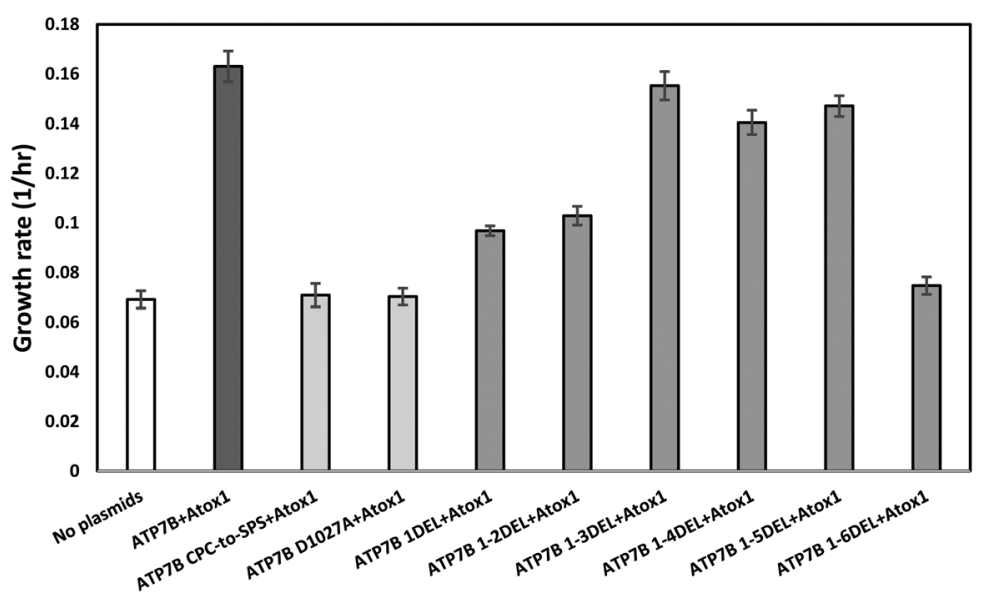

Fig. 3 Growth rates of the $\Delta C C C 2 \Delta$ Atx1 yeast strain complemented with wild type Atox1 plasmid and the various ATP7B variants, as indicated, in Fe-limited conditions. Error bars are based on standard deviation for duplicate experiments. Growth rate values reported in Table S4 (ESI $\dagger$ ).

highly conserved from yeast to humans. Previous work using this approach has provided insights on ATP7B disease-causing mutations as well as putative roles of the six MBDs in ATP7B. ${ }^{13,29-31}$ However, all these studies have been interpreted independently of the $\mathrm{Cu}$ chaperone as only the yeast CCC2 gene was the target of complementation. In the light of the limited knowledge of what domain in ATP7B receives $\mathrm{Cu}$ from Atox1, together with the reports stating that Atox1-MBD interactions are sensitive to mutations and solution conditions and may be of only transient nature, we decided to create a more sensitive system, including exchanging the yeast $\mathrm{Cu}$ chaperone for the human variant. In analogy to our system for studies of human 
Table 1 Relative growth rates of ATP7B variants in the $\Delta C C C 2 \Delta$ Atx1 yeast strain (Fig. 2 and 3). The growth rate of the deletion strain alone $(\Delta C C C 2 \Delta A t \times 1)$ is set to $0 \%$ and the growth of the yeast strain supplemented with both wild type plasmids ( $\triangle \mathrm{CCC} 2 \Delta \mathrm{Atx} 1+\mathrm{ATP7B}+\mathrm{Atox} 1)$ is set to $100 \%$. Only wild type Atox1 was used here

\begin{tabular}{lllc}
\hline ATP7B variant & Atox1 & MBD presence & Relative growth rates (\%) \\
\hline No & No & None & 0 \\
Wild type & No & MBDs 1-6 & 4 \\
No & Yes & None & 6 \\
Wild type & Yes & MBDs 1-6 & 100 \\
ATP7B 1-6DEL & Yes & No MBDs & 6 \\
ATP7B 1-5DEL & Yes & MBD 6 & 83 \\
ATP7B 1-4DEL & Yes & MBDs 5 and 6 & 76 \\
ATP7B 1-3DEL & Yes & MBDs 4-6 & 92 \\
ATP7B 1-2DEL & Yes & MBDs 3-6 & 35 \\
ATP7B 1DEL & Yes & MBDs 2-6 & 30 \\
ATP7B CPC-to-SPS & Yes & MBDs 1-6 & 2 \\
ATP7B D1027A & Yes & MBDs 1-6 & 1
\end{tabular}

$\mathrm{Cu}$ transport presented here, a yeast complementation system was reported recently for analysis of yeast Atx1p and CCC2p variants using dual gene deletions in combination with two rescue plasmids with variants of the yeast genes. ${ }^{25}$

We have here demonstrated that the double deletion complementation yeast system works and we used it to assess the performance of strategic ATP7B variants with focus on the MBDs. From our results on ATP7B constructs with stepwise (one-by-one from the N-terminal) MBD removals, we concluded that the presence of MBD1 makes ATP7B Cu transport more efficient as, without it (ATP7B 1DEL), yeast growth on iron-limited medium was dramatically reduced. No change was found in growth parameters when also MBD2 was removed from ATP7B, however, when three domains, MBD1-3, were removed from ATP7B, wild-type like growth was restored. This can be interpreted as that the presence of MBD3 limits ATP7B-mediated $\mathrm{Cu}$ transport, whereas MBD1in ATP7B promotes ATP7B-mediated $\mathrm{Cu}$ transport such that these effects might cancel out in the full-length protein, perhaps via MBD1-MBD3 interactions. In support, in a recently reported in vitro NMR study exploiting nanobody binding to multi-MBD constructs, transient interactions between MBD1, MBD2 and MBD3 were demonstrated. ${ }^{12}$ Because yeast with the wild-type ATP7B plasmid had a similar growth rate as yeast supplied with ATP7B 1-3DEL, ATP7B 1-4DEL, and ATP7B 1-5DEL, it appears that MBD4 and MBD5 do not modulate ATP7Bmediated $\mathrm{Cu}$ transfer speed per se.

We observed the same effects of MBD truncations on yeast growth regardless of using human Atox1 or Atx1p as the $\mathrm{Cu}^{-}$ delivering chaperone. This suggests that the MBD truncations modulate internal ATP7B processes and not the Cu-receiving step or, at least, that $\mathrm{Cu}$ delivery from the chaperone is not the rate-limiting step in the case of any of these ATP7B variants. It is likely that domain-domain interactions among MBDs and between MBDs and the ATP-binding domain, all putatively modulated by $\mathrm{Cu}$ and ATP binding, contribute collectively to the overall activity of wild-type ATP7B. Thus, one or several of these internal interactions may be affected by the various MBD truncations resulting in the observed pattern of decreased growth. To explain the findings in molecular terms, separation of ATP hydrolysis and $\mathrm{Cu}$ transport kinetic parameters as a function of purified variants of ATP7B will be required.

We also revealed that removal of all six MBDs in ATP7B resulted in an Atox1-ATP7B system that could not promote $\Delta \mathrm{CCC} 2 \Delta \mathrm{Atx} 1$ yeast growth on iron-limited media. This finding was also noted previously for truncated ATP7B in a $\triangle \mathrm{CCC} 2$ strain, but without considering the role of human Atox1 versus Atx1p. ${ }^{13}$ Our result with $\Delta \mathrm{CCC} 2 \Delta \mathrm{Atx} 1$ yeast strongly argues that Atox1 delivers $\mathrm{Cu}$ to one of the MBDs in ATP7B and, unlike in bacteria, ${ }^{24}$ this step cannot be by-passed in the human $\mathrm{Cu}$ transport system.

In similarity to our result presented here, deletion of both MBDs in the CCC2 gene on a plasmid complemented to a $\triangle \mathrm{CCC} 2$ yeast strain resulted in no $\mathrm{Cu}$ transfer by the variant CCC2p. ${ }^{25}$ Additionally, mutating all $\mathrm{Cu}$ sites in the six MBDs (Cys to Ser mutations blocking Cu binding) in the ATP7B gene resulted in non-functional $\mathrm{ATP} 7 \mathrm{~B}$, whereas retaining the Cys residues in MBD6 but not in MBD1-5 restored $\mathrm{Cu}$ transport in yeast at iron-limited conditions. ${ }^{29}$ However, although MBD6 appeared sufficient for function it was not essential as an ATP7B construct with intact $\mathrm{Cu}$ sites in MBD1-5 but with Cys-to-Ser mutations in MBD6 also was found to facilitated $\mathrm{Cu}$ transport at iron-limited conditions when complemented to a $\triangle \mathrm{CCC} 2$ yeast strain. ${ }^{13}$ Clearly, an intricate network of (yet unknown) internal interactions is at play during ATP7B's catalytic cycle.

Looking ahead, the developed yeast complementation system can be used to probe consequences of Cu-site mutations (CXXC to SXXS instead of domain deletions), strategic mutations in Atox1 and in ATP7A (by exchanging the ATP7B gene for the ATP7A gene), and to perform unbiased random mutations to discover gain of function variants of both Atox1 and ATP7A/B. In addition to dead/ live cells and yeast growth rates used here, a range of more advanced and specific functional output parameters can be explored, such as yeast chronological life span, oxidative damage, organelle function, protein content and Fet3p activity. ${ }^{35}$ In extension, we envisage this approach to be valuable for studies directed towards the emerging role of $\mathrm{Cu}$ transport proteins in cancer and neurodegeneration.

\section{Acknowledgements}

We thank the Knut and Alice Wallenberg foundation, the Swedish Research Council and the Chalmers Foundation for funding to PWS and the Novo Nordisk Foundation Center for Biosustainability to DP. We thank Dr A Koc (Izmir Institute of Technology, Turkey) for the ATP7B gene.

\section{References}

1 D. L. Huffman and T. V. O'Halloran, Function, structure, and mechanism of intracellular copper trafficking proteins, Annu. Rev. Biochem., 2001, 70, 677-701.

2 S. Puig and D. J. Thiele, Molecular mechanisms of copper uptake and distribution, Curr. Opin. Chem. Biol., 2002, 6, 171-180. 
3 E. D. Harris, Basic and clinical aspects of copper, Crit. Rev. Clin. Lab. Sci., 2003, 40, 547-586.

4 M. Valko, H. Morris and M. T. Cronin, Metals, toxicity and oxidative stress, Curr. Med. Chem., 2005, 12, 1161-1208.

5 T. V. O'Halloran and V. C. Culotta, Metallochaperones, an intracellular shuttle service for metal ions, J. Biol. Chem., 2000, 275, 25057-25060.

6 R. A. Festa and D. J. Thiele, Copper: an essential metal in biology, Curr. Biol., 2011, 21, R877-R883.

7 N. J. Robinson and D. R. Winge, Copper metallochaperones, Annu. Rev. Biochem., 2010, 79, 537-562.

8 H. Ohrvik and D. J. Thiele, How copper traverses cellular membranes through the mammalian copper transporter 1, Ctr1, Ann. N. Y. Acad. Sci., 2014, 1314, 32-41.

9 S. Lutsenko, N. L. Barnes, M. Y. Bartee and O. Y. Dmitriev, Function and regulation of human copper-transporting ATPases, Physiol. Rev., 2007, 87, 1011-1046.

10 E. S. LeShane, U. Shinde, J. M. Walker, A. N. Barry, N. J. Blackburn, M. Ralle and S. Lutsenko, Interactions between copper-binding sites determine the redox status and conformation of the regulatory N-terminal domain of ATP7B, J. Biol. Chem., 2010, 285, 6327-6336.

11 N. M. Hasan, A. Gupta, E. Polishchuk, C. H. Yu, R. Polishchuk, O. Y. Dmitriev and S. Lutsenko, Molecular events initiating exit of a copper-transporting ATPase ATP7B from the trans-Golgi network, J. Biol. Chem., 2012, 287, 36041-36050.

12 Y. Huang, S. Nokhrin, G. Hassanzadeh-Ghassabeh, C. H. Yu, H. Yang, A. N. Barry, M. Tonelli, J. L. Markley, S. Muyldermans, O. Y. Dmitriev and S. Lutsenko, Interactions between metal-binding domains modulate intracellular targeting of $\mathrm{Cu}(\mathrm{I})$-ATPase $\mathrm{ATP} 7 \mathrm{~B}$, as revealed by nanobody binding, J. Biol. Chem., 2014, 289, 32682-32693.

13 J. R. Forbes, G. Hsi and D. W. Cox, Role of the copperbinding domain in the copper transport function of ATP7B, the P-type ATPase defective in Wilson disease, J. Biol. Chem., 1999, 274, 12408-12413.

14 L. Banci, I. Bertini, F. Cantini, C. Massagni, M. Migliardi and A. Rosato, An NMR study of the interaction of the $\mathrm{N}$-terminal cytoplasmic tail of the Wilson disease protein with copper(I)-HAH1, J. Biol. Chem., 2009, 284, 9354-9360.

15 L. Banci, I. Bertini, F. Cantini, A. C. Rosenzweig and L. A. Yatsunyk, Metal binding domains 3 and 4 of the Wilson disease protein: solution structure and interaction with the copper(I) chaperone HAH1, Biochemistry, 2008, 47, 7423-7429.

16 D. Achila, L. Banci, I. Bertini, J. Bunce, S. Ciofi-Baffoni and D. L. Huffman, Structure of human Wilson protein domains 5 and 6 and their interplay with domain 4 and the copper chaperone HAH1 in copper uptake, Proc. Natl. Acad. Sci. U. S. A., 2006, 103, 5729-5734.

17 A. K. Wernimont, D. L. Huffman, A. L. Lamb, T. V. O'Halloran and A. C. Rosenzweig, Structural basis for copper transfer by the metallochaperone for the Menkes/ Wilson disease proteins, Nat. Struct. Biol., 2000, 7, 766-771.

18 R. A. Pufahl, C. P. Singer, K. L. Peariso, S. J. Lin, P. J. Schmidt, C. J. Fahrni, V. C. Culotta, J. E. Penner-Hahn and
T. V. O'Halloran, Metal ion chaperone function of the soluble Cu(I) receptor Atx1, Science, 1997, 278, 853-856.

19 L. Banci, The Atx1-Ccc2 complex is a metal-mediated proteinprotein interaction, Nat. Chem. Biol., 2006, 2, 367-368.

20 L. Banci, I. Bertini, V. Calderone, N. Della-Malva, I. C. Felli, S. Neri, A. Pavelkova and A. Rosato, Copper(I)-mediated protein-protein interactions result from suboptimal interaction surfaces, Biochem. J., 2009, 422, 37-42.

21 M. S. Niemiec, A. P. Dingeldein and P. Wittung-Stafshede, Enthalpy-entropy compensation at play in human copper ion transfer, Sci. Rep., 2015, 5, 10518.

22 M. S. Niemiec, C. F. Weise and P. Wittung-Stafshede, In vitro thermodynamic dissection of human copper transfer from chaperone to target protein, PLoS One, 2012, 7, e36102.

23 T. Mondol, J. Aden and P. Wittung-Stafshede, Copper binding triggers compaction in N-terminal tail of human copper pump АТР7B, Biochem. Biophys. Res. Commun., 2016, 470, 663-669.

24 M. Gonzalez-Guerrero and J. M. Arguello, Mechanism of $\mathrm{Cu}$-transporting ATPases: soluble $\mathrm{Cu}+$ chaperones directly transfer $\mathrm{Cu}+$ to transmembrane transport sites, Proc. Natl. Acad. Sci. U. S. A., 2008, 105, 5992-5997.

25 I. Morin, S. Gudin, E. Mintz and M. Cuillel, Dissecting the role of the N-terminal metal-binding domains in activating the yeast copper ATPase in vivo, FEBS J., 2009, 276, 4483-4495.

26 A. Rodriguez-Granillo, A. Crespo and P. Wittung-Stafshede, Interdomain interactions modulate collective dynamics of the metal-binding domains in the Wilson disease protein, J. Phys. Chem. B, 2010, 114, 1836-1848.

27 L. Nilsson, J. Aden, M. S. Niemiec, K. Nam and P. WittungStafshede, Small $\mathrm{pH}$ and salt variations radically alter the thermal stability of metal-binding domains in the copper transporter, Wilson disease protein, J. Phys. Chem. B, 2013, 117, 13038-13050.

28 D. Huster, A. Kuhne, A. Bhattacharjee, L. Raines, V. Jantsch, J. Noe, W. Schirrmeister, I. Sommerer, O. Sabri, F. Berr, J. Mossner, B. Stieger, K. Caca and S. Lutsenko, Diverse functional properties of Wilson disease ATP7B variants, Gastroenterology, 2012, 142, 947-956, e945.

29 M. A. Cater, J. Forbes, S. La Fontaine, D. Cox and J. F. Mercer, Intracellular trafficking of the human Wilson protein: the role of the six N-terminal metal-binding sites, Biochem. J., 2004, 380, 805-813.

30 M. Iida, K. Terada, Y. Sambongi, T. Wakabayashi, N. Miura, K. Koyama, M. Futai and T. Sugiyama, Analysis of functional domains of Wilson disease protein (ATP7B) in Saccharomyces cerevisiae, FEBS Lett., 1998, 428, 281-285.

31 G. Hsi, L. M. Cullen, G. Macintyre, M. M. Chen, D. M. Glerum and D. W. Cox, Sequence variation in the ATP-binding domain of the Wilson disease transporter, ATP7B, affects copper transport in a yeast model system, Hum. Mutat., 2008, 29, 491-501.

32 A. Froger and J. E. Hall, Transformation of plasmid DNA into $E$. coli using the heat shock method, J. Visualized Exp., 2007, 6, 253. 
33 A. Wach, A. Brachat, R. Pohlmann and P. Philippsen, New heterologous modules for classical or PCR-based gene disruptions in Saccharomyces cerevisiae, Yeast, 1994, 10, 1793-1808.

34 H. Y. Steensma and J. J. Ter Linde, Plasmids with the Crerecombinase and the dominant nat marker, suitable for use in prototrophic strains of Saccharomyces cerevisiae and Kluyveromyces lactis, Yeast, 2001, 18, 469-472.

35 O. S. Papur, O. Terzioglu and A. Koc, Functional characterization of new mutations in Wilson disease gene (ATP7B) using the yeast model, J. Trace Elem. Med. Biol., 2015, 31, 33-36.

36 R. D. Gietz and R. A. Woods, Yeast transformation by the Li/Ac/SS carrier DNA/PEG method, in Yeast Protocol, ed. W. Xiao, Humana Press, Totowa, NJ, 2006, DOI: 10.1385/ 1-59259-958-3:107, pp. 107-120.

37 M. Andersson, D. Mattle, O. Sitsel, T. Klymchuk, A. M. Nielsen, L. B. Moller, S. H. White, P. Nissen and P. Gourdon, Coppertransporting P-type ATPases use a unique ion-release pathway, Nat. Struct. Mol. Biol., 2014, 21, 43-48. 